\title{
Blockchain based Big Data Platform of City Brain
}

\author{
Junfang Zeng \\ Institute of Automation, Chinese Academy of Sciences \\ junfang.zeng@ia.ac.cn
}

\begin{abstract}
Blockchain technology, with its distributed ledger, decentralization, high security, no tampering and other features, helps to solve the problems of data source confirmation, traceability and authorized data sharing. The effective utilization of data to achieve automatic governance and trusted decision-making of the city is known as "City Brain". The city brain is the key in smart city development, while the key to build a city brain is data resources. Aiming at the challenges existing in the construction of big data platform, this paper proposes a new solution based on blockchain, establishes the entity model and data model, analyzes the business model, designs the blockchain data platform framework and the cloudblockchain integrated operation mode, and at last discusses the issues concerned in the application.
\end{abstract}

\section{CCS CONCEPTS}

- Blockchain; • Big data sharing; • Multicentric;

\section{KEYWORDS}

Cloud-blockchain integrated, Index on blockchain, Smart contract, City brain

\section{ACM Reference Format:}

Junfang Zeng and Yu Liu. 2021. Blockchain based Big Data Platform of City Brain. In 2021 The 3rd International Conference on Blockchain Technology (ICBCT '21), March 26-28, 2021, Shanghai, China. ACM, New York, NY, USA, 8 pages. https://doi.org/10.1145/3460537.3460561

\section{INTRODUCTION}

With the development of artificial intelligence (AI), big data and cloud computing, government information openness, data sharing and cross sector linkage become the trend in the construction of city brain, "data" has become a digital assert. The city brain is required to connect the data scattered in all corners of the city, through the analysis and integration of a large amount of data, it conducts real-time analysis, predict, scheduling and management of the city, to achieve accurate analysis, overall judgment, and collaborative command. However, at present the city big data platform mostly adopts a centralized mode, data gathers to the data center and the data request is directly submitted to the centralized system. It is difficult to go through multiple business fields and information

Permission to make digital or hard copies of all or part of this work for personal or classroom use is granted without fee provided that copies are not made or distributed for profit or commercial advantage and that copies bear this notice and the full citation on the first page. Copyrights for components of this work owned by others than ACM must be honored. Abstracting with credit is permitted. To copy otherwise, or republish, to post on servers or to redistribute to lists, requires prior specific permission and/or a fee. Request permissions from permissions@acm.org.

ICBCT '21, March 26-28, 2021, Shanghai, China

(C) 2021 Association for Computing Machinery.

ACM ISBN 978-1-4503-8962-4/21/03 . \$15.00

https://doi.org/10.1145/3460537.3460561

\author{
Yu Liu \\ Institute of Automation, Chinese Academy of Sciences \\ yu.liu@ia.ac.cn
}

systems due to large amount of data and huge cost, no ensuring that the data is reliable, accurate and transparent, resulting in the data application is still in a low online rate and utilization rate, and it does not fully take advantage of big data, and the technology used in the platform cannot keep up with the speed of data growth. Blockchain technology $[1,4]$ helps to provide a systematic solution for big data sharing.

Blockchain technology is adoptable for decentralized application scenarios with multi-party participation, common maintenance to enhance trust [3, 6-7]. For the utilization of government public data, there are scenarios well matching the features of blockchain technology. The combination of data sharing and blockchain technology, the no tampering and traceability capabilities of blockchain, solves the problems of data openness and data security, and realizes the pluralism between data chains and the pluralism of data using entities. Data island, unclear data ownership and data monopoly are still the difficulties in data sharing. Blockchain provides a new solution to solve the problems in government data opening, data security and data ownership. The decentralized feature of blockchain enables multi parties to cooperate together. Under the specific management rules (consensus mechanism [2]), multi parties can monitor and manage complete data in the distributed ledger together, to realize the "multiparty co-governance" of data.

This paper researches on blockchain based multicentric big data sharing, designs the framework and cloud-blockchain integrated mechanism, which stores and process data in a distributed multi center way. Based on the unified management of shared data, only authorized users can access the shared data by using smart contract mechanism [5]. Through hierarchical permission management, the data is classified and graded for fine-grained sharing, to ensure the balance between data security and its openness.

\section{BIG DATA SHARING MODELING}

\subsection{Entity model}

The city big data platform involves participates (called "entity") from all levels of the administrative organization structure, and they have different functions, privacy and interests. To build a blockchain application system, we need first establish the entity model, analyze the attributes and behaviors of each entity, and the interaction between them. The entity model of blockchain big data platform is shown in Figure 1

The participants of blockchain big data platform include government sectors, Big Data Bureau, enterprises/institutions, public, big data development team and more. The business process of big data platform includes data production, processing and usage [8], involving multiple entities. The data collected by the government sectors and other units through information systems and IOT devices [13] belongs to the entity itself, who holds the ownership and uploads the data according to government regulatory requirements. 


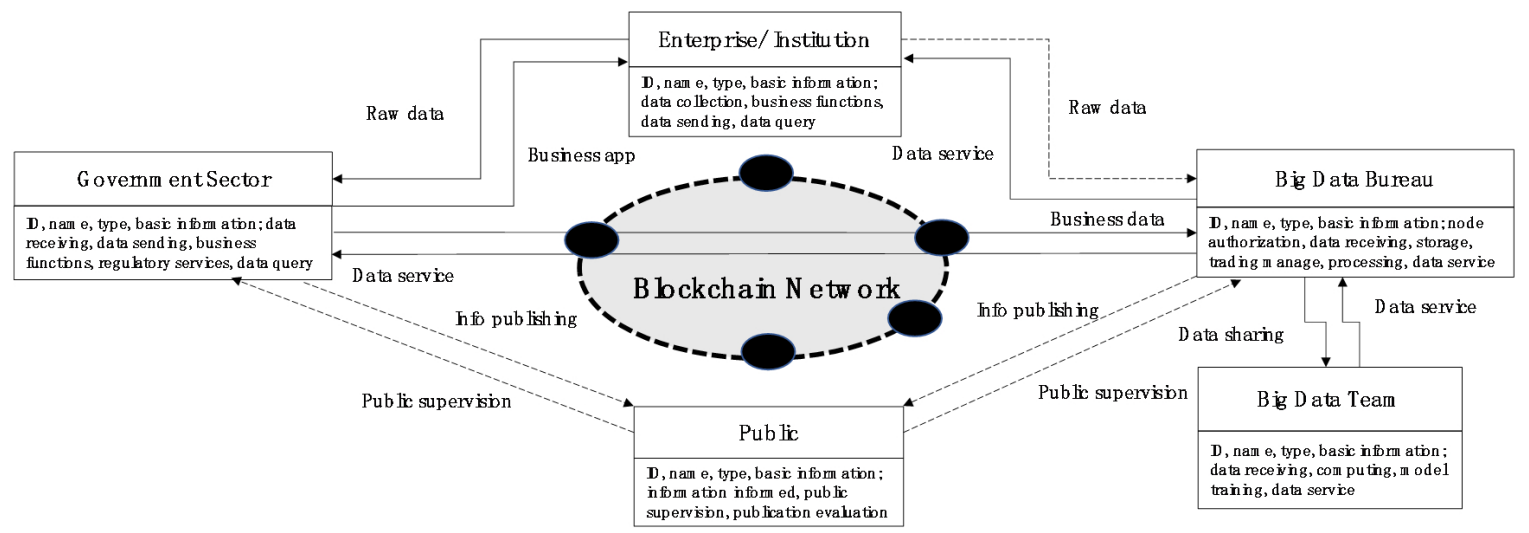

Figure 1: Entity model of blockchain based big data platform.

The city Big Data Bureau stores, processes and pushes the collected data to the relevant sectors, who is not only the user of data, but also the provider of processing data; the government sector obtains shared data from other government sectors, enterprises and institutions, who is the user of processing data; big data integration, data analytics and mining, data utilization and reuse, such as analysis, statistics, predict and decision-making based on shared data [16], and the professional development team is the service provider of data analytics. Each sector uses shared data to provide application services in its own business fields, such as energy conservation regulatory services provided by energy conservation authorities.

Three tuples are used to express the entity: Entity $=<$ Attribute, Behavior, Relation $>$, where Attribute is the ID, name, type, basic information, digital signature, etc. of the entity; Behavior is the data processing and business functions executed by the entity; Relation is the interaction between entities, including input, output and trading behavior.

\subsection{Data model}

Blockchain technology runs through the whole life cycle of data [15]. Blockchain stores a record of data trading (data interaction between entities) in the form of a decentralized ledger, links data distributed in different storage blocks according to timestamp. Data trading records are transparent and traceable to participants. The source, flow path and access of data are recorded and traced. Every update and modification of data could be marked. At the same time, hash algorithm is used to verify the integrity of data and prevent data from being tampered, to ensure and improve the quality of data in sharing.

The data model of the big data platform is established by interacting the relevant data among the participants of the blockchain, as shown in Figure 2, including Entity info, Metadata, Directory data and Trading data.

The shared data of big data platform involves private data, and security guarantee is required in data collection, transmission, storage, processing and usage. Hierarchical authority management is adopted, different sectors and different levels of users could access different data; data is stored in each node of the blockchain and synchronized in real time, avoiding to report data layer by layer; data distributed deployment realizes high concurrency, high stability and high scalability.

Besides business data in the database, additional information such as description text, file, picture, voice, video, evaluation, rules and so on could be stored in IPFS (Interplanetary File System). The index of shared data computed by hash algorithm, added with timestamp and digital signature of the provider, is stored on the blockchain [18], and connected with relevant smart contracts for data query and automatic access.

\subsection{Business model}

The trading data of e-government data, traffic data, energy and power data, medical data, industrial data and other fields are stored on blockchain, automatically form a decentralized ledger which manages the ledger data across multiple nodes. Based on the entity model and data trading model, we analyze and plan the data processing logic of the big data platform, as shown in Figure 3

- The shared data generated by the application systems of government bureaus are synchronized to the front-end unit of each local node;

- The data is packaged and uploaded to the platform by the front-end unit, then processed, granulated and classified, stored in structured raw DB, directory DB and subject DB (data on cloud);

- At the same time, the data is computed hash algorithm by the front-end unit, and the timestamp and digital signature are added to generate the hash index of the data, which is saved to the directory index blockchain of the platform (index on blockchain);

- The shared data of the platform are provided to the sectors, enterprises, units, etc. through APIs, and the open information is released to the public. It is also used by the big data development team who use AI algorithms to make statistics, prediction and decision on the reliable and secured history data.

- After data aggregation from multiple repositories, use AI technology to mine data value, establish data-driven decision-making mechanism. It supports data integration between index on the chain and raw data under the chain, 


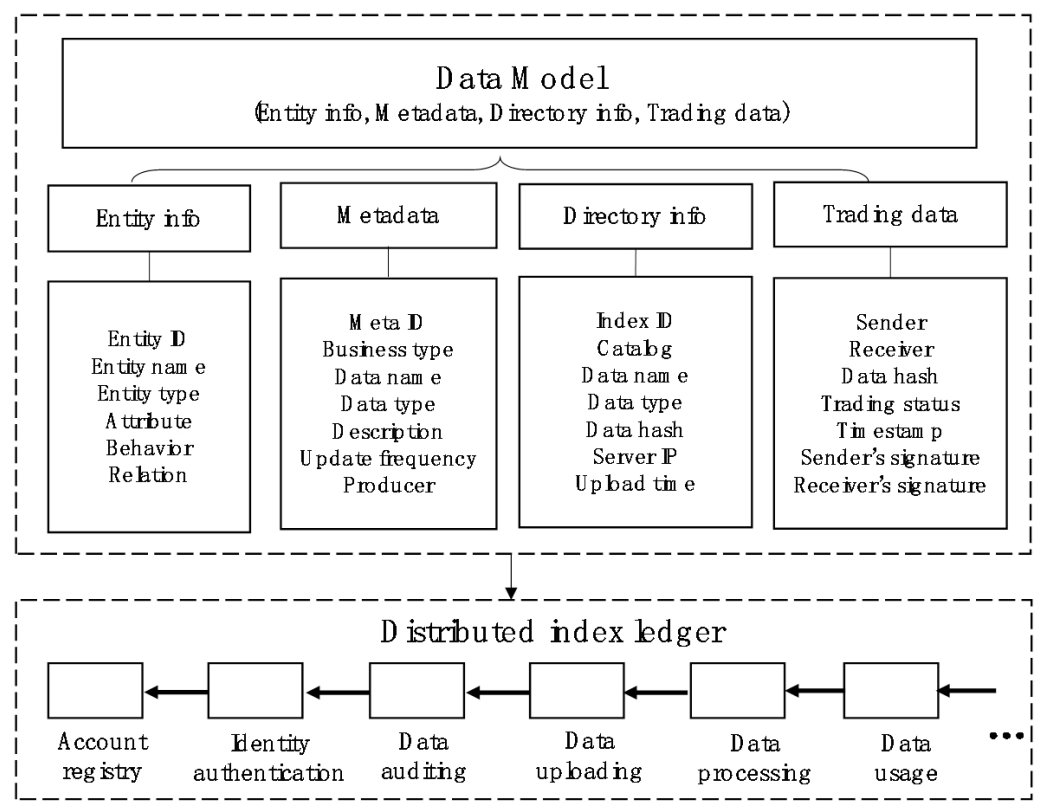

Figure 2: Data model of blockchain based big data platform.

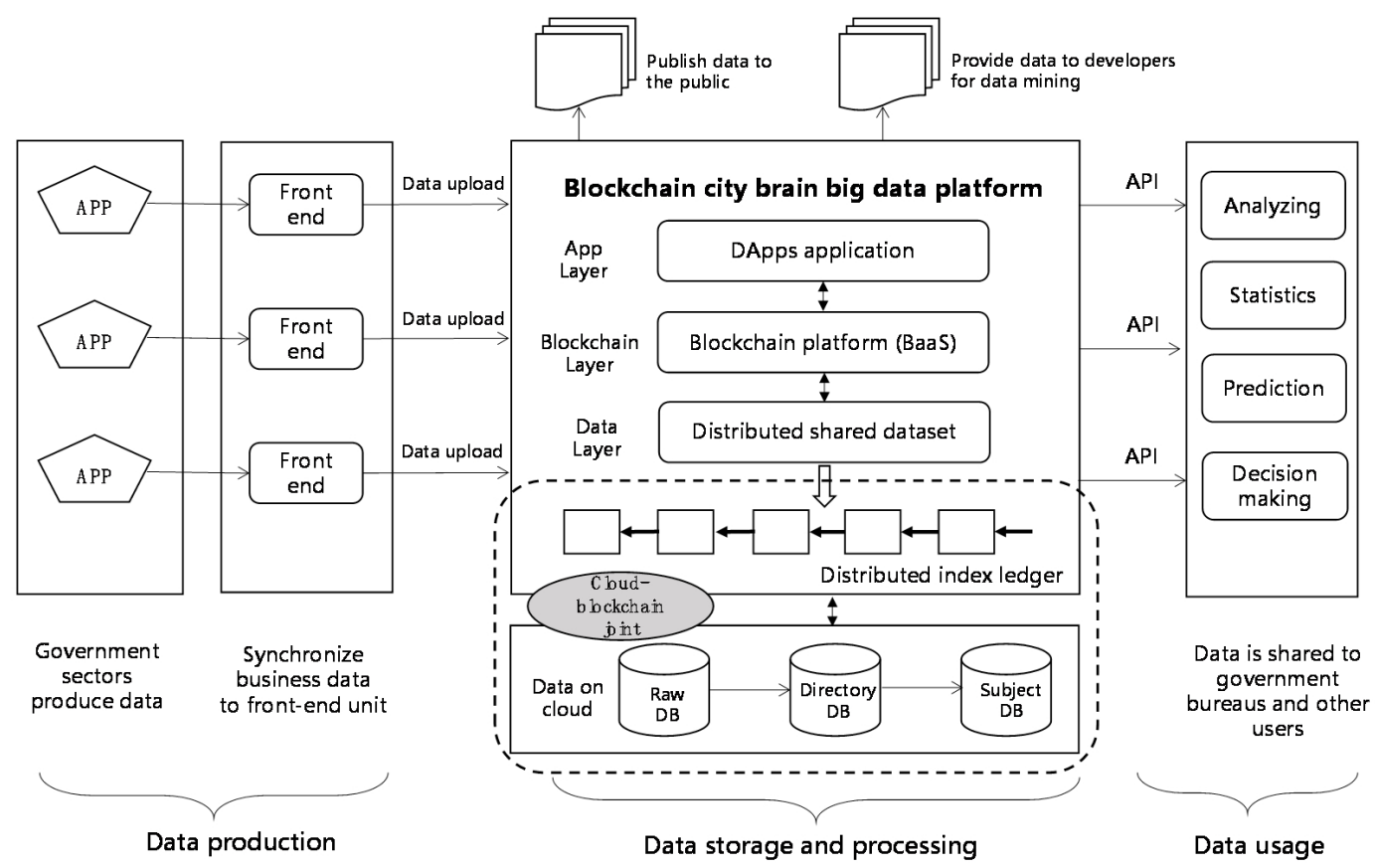

Figure 3: Business process logic diagram.

solving the problem of data openness on the premise of protecting user privacy.

\subsection{Smart contracts}

Data trading of big data platform is automatically executed through the smart contract mechanism of blockchain. Smart contracts are coded and published to blockchain for execution under specific circumstances. We roughly divided them into four categories: management contract, data contract, computing contract and service contract. Each type of smart contract could be further subdivided, as shown in Figure 4 


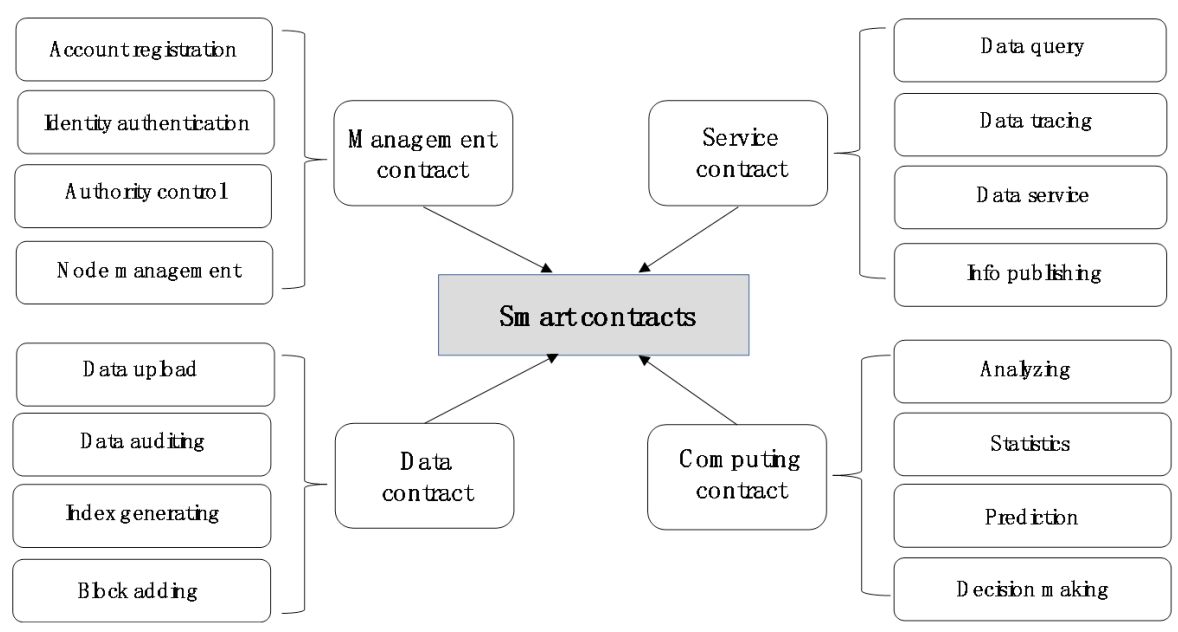

Figure 4: Composition of smart contracts of big data platform.

Considering that there is a certain trust relationship between the parties in the city brain big data platform, it is appropriate to use the consortium blockchain mode to build it [14, 17], and the nodes join and exit the blockchain network through authorization. Participants of the blockchain create smart contracts (write a script) and register contract accounts for publishing. Verification nodes audit smart contracts and confirm the effectiveness of the contracts under the consensus mechanism. The effective contract uses the APIs provided by the platform to automate the smart contract execution. Each time a user accesses data, he initiates a trading and a specific smart contract is automatically executed in the application.

\section{FRAMEWORK AND OPERATION MODE}

\subsection{Framework of data sharing platform}

The city brain is built to realize five main functions of city operation, emergency response, public security, decision-making and services, focusing on data-based perception, analysis, predict, scheduling and judgment. The framework of blockchain big data platform is shown in Figure 5, with five layers from bottom to top: blockchain data layer, blockchain core layer, blockchain interface layer, decentralized application layer and presentation layer [9-10]. Based on the underlying platform of blockchain, the smart contracts and incentive mechanism are customized based on business model, and the decentralized application DAPPs in various business fields are developed, covering the whole business process of city brain big data platform.

- 1. Blockchain data layer: provides business blockchains (egovernment chain, transportation chain, environment chain, energy chain and so on) including metadata, directory info, trading data, tracking data. Multi signature permission control is used to ensure data security.

- 2. Core layer of blockchain: supported by the technology of consortium blockchain, it provides account management, privacy protection, distributed ledger, smart contracts, consensus mechanism, token mechanism, etc.; provides management services of authorization, synchronization, data storage, data trading and data query.

- 3. Blockchain interface layer: provides APIs for blockchain application development, supports account creation, identity authentication, data auditing, data upload, data trading, data query, etc.

- 4. Business application layer: based on the collected data of blockchain big data platform, the decentralized applications of government sectors and users run on the business blockchains, including e-government DAPP, transportation DAPP, environment DAPP, energy DAPP, etc.

- 5. HMI layer: user interface to present the results of data analytics in a visual way. Users include government sectors, enterprises, institutions, big data development teams and the public. Web version of B/S structure and mobile APP version are provided.

\subsection{Cloud-blockchain integrated mechanism}

The cloud-blockchain integrated mechanism is adopted in the city brain big data platform, to achieve "data on cloud, index on blockchain". It not only takes advantage of cloud: huge storage, super computing power and unified management, but also makes use of the features of blockchain: data fidelity, full transparency, traceability, no tampering, easy expansion and cross entity collaboration. Different application scenarios of data sharing could form different data streams through blockchain. When it is shared between multiple parties, the data granularity management should be carried out accurately and quickly according to different needs of users (combination of roles).

Role access control could clarify the data source, data ownership, data modification path and block location when the data is finally disclosed; the accuracy of data is not only reflected in the accuracy of the data itself, but also includes all records of data value and data life cycle, which could accurately describe the shared data and information. At the same time, due to the decentralized features 


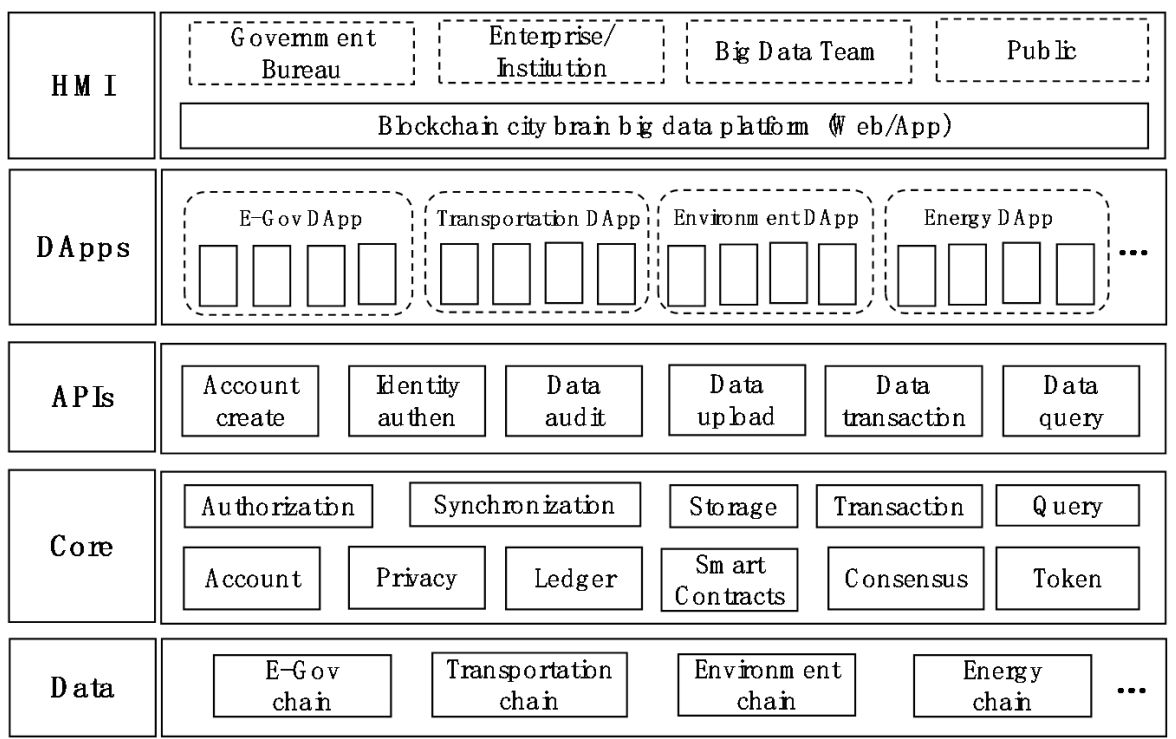

Figure 5: Framework of blockchain based city brain big data platform.

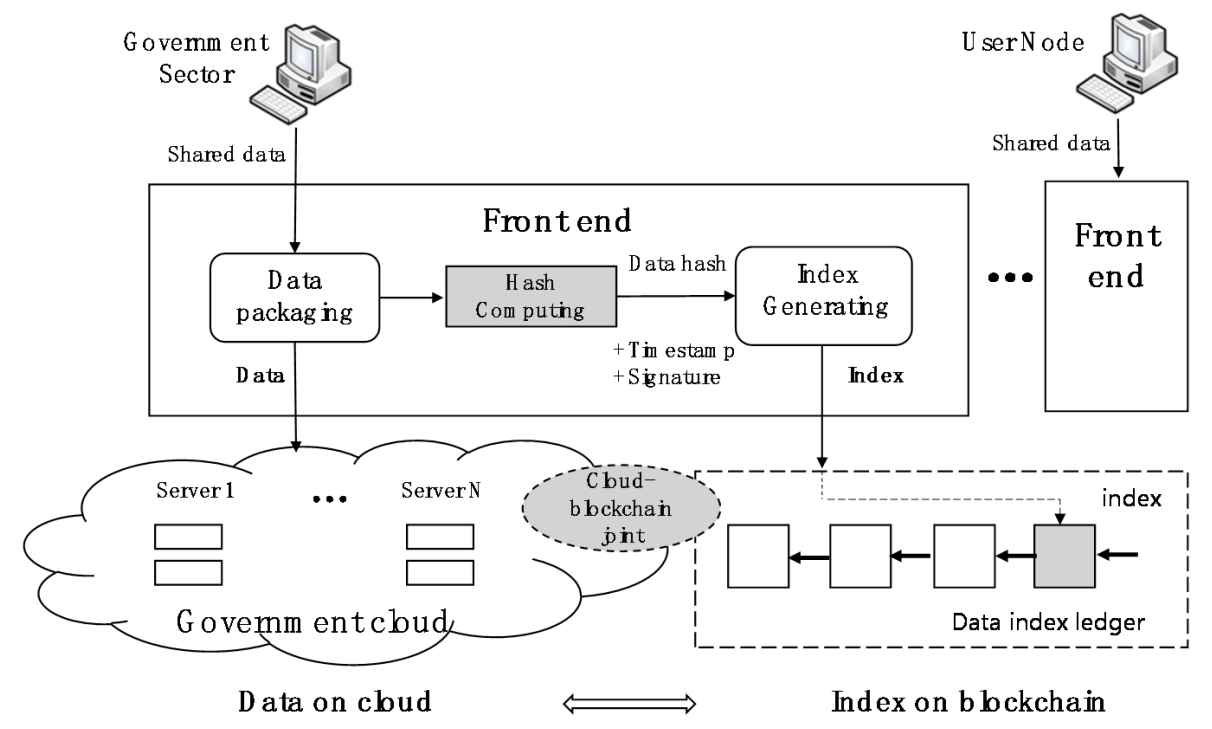

Figure 6: Cloud-blockchain integrated mechanism of city brain big data platform.

of blockchain technology, the database will not be attacked or tampered to cause the data link collapse. Different access rules are given to the massive data groups gathered on the platform, and different data combinations are obtained according to different requirements of each entity through the smart contract mechanism of blockchain. The city brain uses the datasets on cloud indexed by blockchain multicentric ledger, as shown in Figure 6

\subsection{Operation mode}

- Data trading of city big data sharing platform is automatically executed through the smart contract mechanism of blockchain. Smart contracts are coded and published to blockchain, executed under specific circumstances. In deploying blockchain applications, the smart contract-driven operation mode is designed for the blockchain based city big data platform, as shown in Figure 7. The process of data sharing and business linkage is as follows:

- 1. The front-end unit of each government bureau and user node is registered, and the registration information is sent to the blockchain network. After verification, it becomes a legal node (account management contract); 


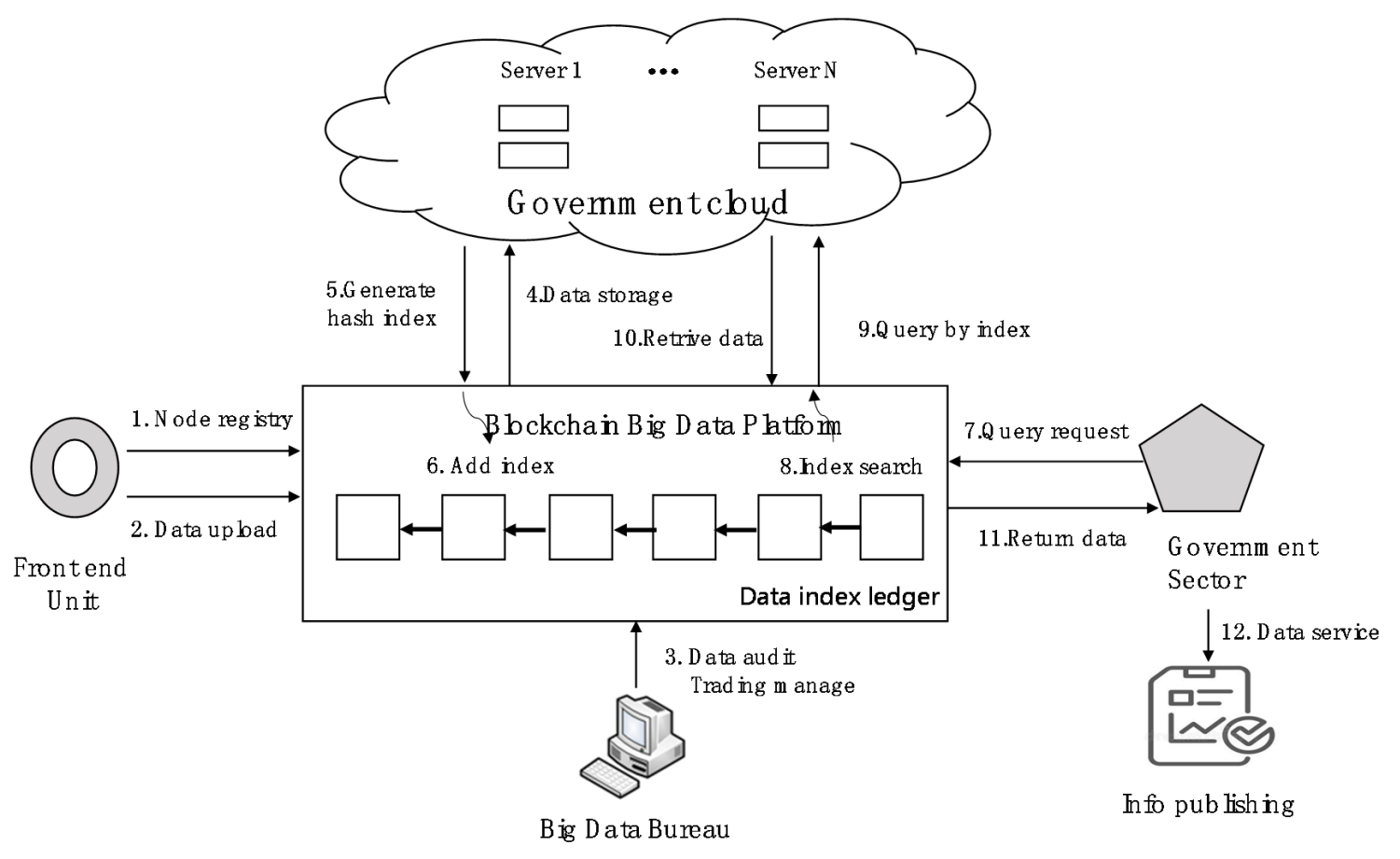

Figure 7: Operation mode of blockchain based city brain big data platform.

- 2. Each government bureau and user node synchronize the produced business data to its front-end unit by which data is uploaded to the platform (data upload contract);

- 3. The city Big Data Bureau checks the validity of the shared data (data audit contract) and takes the role of data management (data management contract);

- 4. The approved data is packaged and uploaded by the frontend unit, then uploaded and stored on government cloud or centralized servers (data storage contract);

- 5. The front-end unit executes the hash algorithm to process the shared data, adds timestamp and digital signature, and generates the hash index of the data (index generating contract);

- 6. The big data platform packages and encrypts the hash index of shared data, and stores it on the blockchain (index on blockchain contract);

- 7. The government bureau and user node input data query request to the platform to retrieve the required shared data (data query contract);

- 8. After verifying the user's authority, the platform reads the hash index of data from the blockchain through the platform's search function (index search contract);

- 9. Retrieve the raw shared data from the government cloud server according to the hash index, and link it to the query record;

- 10. The big data platform generates a response and sends the data trading record to the blockchain network (data trading contract);
- 11. The government bureau and user node obtain the data returned in response to the request and performs comprehensive computing (data computing contract);

- 12. The big data platform outputs the results to government bureaus, enterprises, institutions and the public, and provides various data services (data service contract).

\section{ISSUES CONCERNED IN APPLICATION}

The construction of city brain big data platform is a systematic project with high integrity, involving data sharing and data integration of multiple sectors, businesses, platforms and manufacturers. The application of big database platform is driven by data, excavates data value, realizes business linkage, and runs, manages and maintains the data platform. Some of issues concerned in application are discussed as below.

\subsection{Distributed ledger management}

Blockchain adopts distributed ledger, and multiple verification nodes ("multi center") could be set. Each verification node has complete ledger, that is, full data. Multiple verification nodes synchronize data under consensus mechanism. When the blockchain network generates new trading data, one of the verification nodes packages them and generate a new block, then broadcasts it to the whole network. The other nodes verify whether the new data is valid. After reaching consensus, each node adds this new block to the ledger to complete multi center data maintenance [11-12]. 


\subsection{Data traceability}

If the data received by the big data platform has problems (inaccuracy, low quality, tampering, etc.), it will bring adverse effects to the subsequent data processing and use, resulting in unreliable results. Using blockchain technology, as data in the whole life cycle is transparent and visible, we could trace back all trading on the chain. Starting from the current block, traverse the whole blockchain according to the order of block generation to find out the problem data and the responsible entity.

\subsection{Data privacy issue}

The shared data of each entity includes private data, shared data and open data. The private data is still stored in the database on the Intranet of the entity, the shared data is uploaded according to the requirements of the big data platform, and the desensitized open data is released on the Internet. The blockchain platform with account registration, identity authentication, digital signature, encrypted data transmission and storage, data granularity management, hierarchical authorization and so on helps to protect data privacy.

\subsection{Data usage mark}

Starting from data generation, the shared data is uploaded to the big data platform, and provided to users for utilization. According to the sharing contracts of blockchain, data access control could work on data of different granularity and scope, including the change of data flow direction and management authority. With the features of data fidelity, transparency and anti tampering of blockchain, we could mark the use of data, who used the data, how many times and how long it was used, who is responsible for it, and all the proof stays auditable.

\subsection{Data security in big data development}

In centralized mode, development team have access to full or part of raw data, under the threat of using the data for other purposes. The blockchain based big data platform holds distributed ledger, external systems connect the platform through APIs, and execute the data acquisition smart contract to access anonymized data (in encrypted format) for application development, AI model training and decision making. The procedure could be done without providing raw data to the team, resulting in improved data security.

\subsection{Insensitive data upload and update}

The blockchain big data platform has good scalability for new data sharing requirements between different businesses and different databases, and the nodes could join and exit at any time. Through the data acquisition smart contract and the APIs provided by the platform, the front-end unit at the node side will upload (according to report frequency or trigged by the abnormal event) and update the trading data in time. This process is data driven and auto fulfilled by smart contract, insensitive to the business application.

\subsection{Impact of using blockchain technology}

Three modes for building big data platform: the traditional data center mode, the blockchain multicentric mode, and the cloudblockchain integrated mode by combining the two, that is, "data on cloud, index on blockchain". The government cloud is still used to manage the shared data, while the index (trading data) is saved on the chain. The cloud-blockchain integrated mode has minimum impact on the existing business of the big data platform. The data processing under the blockchain makes the business proceed as usual, and take advantages of blockchain data fidelity, traceability, tamper proof, and facilitate data retrieval as well.

\section{CONCLUSION}

The city brain is the key in smart city development. It is essential to build a big data platform that gathers data from different distributed data sources, it is challenging to open shared data without loss of privacy. Blockchain technology is an effective approach to achieve data source fidelity, transparency, traceability and data synchronous sharing. In view of the challenges existing in building city brain big data platform, this paper proposes a multicentric solution based on blockchain, which is characterized by front-end synchronous data, five layered framework of the platform, cloud-blockchain integrated mechanism, contract-driven operation mode, etc.

Blockchain technology is becoming the trend of digital economy in the future. The blockchain based city big data platform provides the development opportunity of sharing and co-governance for social governance. Blockchain can provide high quality and effectiveness of data opening from technical level. Through the government's open access interface, data information can be provided to other relevant users in the form of free or appropriate charges. Data users could process the acquired data to achieve new value-added, and further provide it to other users. This process can realize the goal of data sharing open ecology.

The city big data platform based on blockchain is not only the upgrading of the technical level, but also the innovation of management model, which realizes the trusted data collection, on-demand acquisition, safe trading and authorized use, so as to improve the efficiency of data flow, tracking and data utilization, and improve the intelligent and accurate level of city management. In the process of data opening, how can the government define the scope of data opening properly, and protect data privacy while opening data to the public. On the one hand, blockchain technology helps to reconstruct the database, establish a new ecology of urban big data, and improve data accuracy and transparency; on the other hand, blockchain technology can promote data marketization, realize data diversification, and form a value chain of shared data [19]. In the future, with the construction of smart cities all over the world, the big data sharing ecology also includes sharing data of other blockchain platforms. To transfer data assets across blockchains and platforms, it is challenging to realize decentralized and efficient asset trading through cross chain technology. At the same time, the results of big data analytics also need to distribute the digital assets of other blockchain networks or execute cross chain smart contracts in a decentralized way, which requires the city brain big data platform to have the ability of cross chain interaction, posing greater challenges to blockchain technology. 


\section{ACKNOWLEDGMENTS}

This research is supported by National Defense Basic Scientific Research Program (Grant no: JCKY2019203C029).

\section{REFERENCES}

[1] Atul Adya, Paramvir Bahl, Jitendra Padhye, Alec Wolman, and Lidong Zhou. 2004. A multi-radio unification protocol for IEEE 802.11 wireless networks. In Proceedings of the IEEE 1st International Conference on Broadnets Networks (BroadNets'04) . IEEE, Los Alamitos, CA, 210-217. https://doi.org/10.1109/BROADNETS. 2004.8

[2] DWYER G P. The economics of bitcoin and similar private digital currencies[J] Journal of Financial Stability, 2015, 17: 81-91.

[3] BENTOVI, LEEC, MIZRAHIA, et al. Proof of Activity: Extending Bitcoin's Proof of Work via Proof of Stake[J]. Acm Sigmetrics Performance Evaluation Review, 2014, 42(3): 34-37.

[4] LYU Shining. YAN Yong, DING Qi, et a1. Application of blockchain in energy internet: advantage, scenario and case[J]. Zhejiang Electric Power, 2017, 36(3): $1-4$

[5] YUAN Yong, WANG Feiyue. Blockchain: the state of the art and future trends[J]. Acta Automatica Sinica, 2016, 42(4): 481-494.

[6] Christidis, K. and Devetsikiotis, M. Blockchains and smart contracts for the Internet of Things[EB/OL]. [2016-05-10]. IEEE Access Vol.4: 2292-2303.

[7] https://doi.org/10.1109/ACCESS.2016.2566339.

[8] YAN Yong, ZHAO Junhua, WEN Fushuan, et al. Block-chain in energy systems: concept, application and prospect[J]. Electric Power Construction, 2017, 38(2): $12-20$.

[9] YANG Dechang, ZHAO Xiaoyu, XU ZiXiao, et al. Developing status and prospect analysis of blockchain in energy internet[J]. Proceedings of the CSEE, 2017,
37(13): 3664-3671.

[10] Dept. of Environment \& Resources Conservation NDRC, National Energy Conservation Center. Technical specifications for online monitoring of key energy consumption units (Trial), the first publishing of 12 specifications [EB/OL]. [201805-11].

[11] http://www.chinanecc.cn/website/News!view.shtml?id=207786.

[12] Glaser, F. Pervasive decentralisation of digital infrastructures: a framework for blockchain enabled system and use case analysis. 50th Hawaii International Conference on System Sciences (HICSS 2017), Waikoloa, Hawaii, USA, 1-14.

[13] Chen S, Shi R, Ren Z, et al. A blockchain based supply chain quality management framework[C]. 14th IEEE Inter-national Conference on E-Business Engineering (ICEBE 2017), Shanghai, China, 2017:172-176.

[14] YANG Mingtong, ZHOU Buxiang, DONG Shen, et al. De-sign and dispatch optimization of microgrid electricity market supported by blockchain [J]. Electric Power Automation Equipment, 2019, 39(12): 155-161.

[15] MICHAEL C. Ethereum used for 'first' paid energy trade using blockchain tech [EB/OL]. [2016-04-11]. http://www.coind-esk.com/ethereum-used-first-paidenergy-trade-using-blockchain-technology/.

[16] IRINA S W. Block Charge: the blockchain based solution for charging electric cars [EB/OL]. [2016-10-27]. https: //www. 1inkedin. corn/pulse/blockchargeblockchain based-solution charging-ears/.

[17] Buterin, V. On public and private blockchains. Ethereum Blog, Crypto Renaissance Salon. 7th August 2015.

[18] YANG Ting, ZHAO Junjie, ZHANG Weixin, et al. Data blockchain generation algorithm of cyber physical power system[J]. Electric Power Automation Equipment, 2018, 38(10): 80-86.

[19] MA Tiannan, PENG Lilin, DU Ying, et al. Competition game model for local multimicrogrid market based on blockchain technology and its solution algorithm [J]. Electric Power Automation Equipment, 2018, 38 (5): 198-210. 